\title{
Effect of undulators on magnet lattice and emittance
}

\author{
Victor Smaluk@, Yongjun Li, Yoshiteru Hidaka, Toshiya Tanabe®, Oleg Chubar, Lutz Wiegart, \\ Alexei Blednykh $\odot$, Belkacem Bacha, and Timur Shaftan \\ Brookhaven National Laboratory, Upton, New York 11973, USA
}

(Received 16 July 2019; published 17 December 2019)

\begin{abstract}
NSLS-II is now one of the brightest synchrotron light sources worldwide, in user operations since 2014. Currently, 20 light-generating insertion devices (IDs) are installed: 3 damping wigglers and 17 undulators of various types. These devices introduce significant distortions into the magnet lattice of the storage ring. For each ID, the distortions have been locally corrected using quadrupole magnets located nearby the ID. The insertion devices have also a significant impact on the electron beam emittance. Precise measurements of the emittance were carried out at the Coherent Hard X-ray beamline for three different lattices: without IDs, with 3 pairs of damping wigglers only, and with all IDs. A minimum horizontal emittance of $0.76 \mathrm{~nm}$ was achieved. The radiation energy loss and relative energy spread were also measured using a streakcamera. The measured data show good agreement with analytical formulas and numerical simulations.
\end{abstract}

DOI: 10.1103/PhysRevAccelBeams.22.124001

\section{INTRODUCTION}

NSLS-II is one of the brightest synchrotrons in operation. The storage ring consists of 15 mirror-symmetric Double-Bend Achromat supercells with short $(6 \mathrm{~m})$ and long $(9 \mathrm{~m})$ straight sections. To decrease the beam emittance, 3 pairs of damping wigglers are installed. Figure 1 shows beta functions and dispersion for two cells, one with two undulators and the other with two damping wigglers.

The NSLS-II storage ring was commissioned in 2014 with the damping wigglers and six undulators installed as a part of the NSLS-II project. Since the wigglers significantly effect the magnet lattice and beam dynamics, two options for the lattice were developed and optimized during commissioning: the lattice without insertion devices ("bare") and the operational lattice with 3 pairs of damping wigglers (3DW). Effects of six installed undulators were small and therefore not included in the initial lattice model. Table I shows typical NSLS-II lattice parameters calculated for both options.

The 3DW lattice has been used for user operations since 2014. However, more and more undulators were installed in the ring every year, so now NSLS-II operates with 17 undulators of various types. The main parameters of the light-generating insertion devices (IDs) are summarized in

\footnotetext{
"smaluk@bnl.gov
}

Published by the American Physical Society under the terms of the Creative Commons Attribution 4.0 International license. Further distribution of this work must maintain attribution to the author(s) and the published article's title, journal citation, and DOI.
Table II: magnet length $L$, period $\lambda_{p}$, magnet gap, and undulator strength parameter $K$ defined as [1].

$$
K=\frac{c B_{0} \lambda_{p}}{2 \pi E_{e} / e}
$$

where $B_{0}$ is the peak magnetic field and $E_{e}=m_{e} c^{2}$ is the electron rest energy. For every insertion device, the beamline and the year of commissioning are also shown.

\section{COMPENSATION OF OPTICS DISTORTION CAUSED BY UNDULATORS}

Since the total effect of all 17 undulators is not negligible, an updated lattice model has been developed. The wigglers and undulators are modeled as 2-dimensional kickmaps calculated by RADIA Magnetostatics Computer

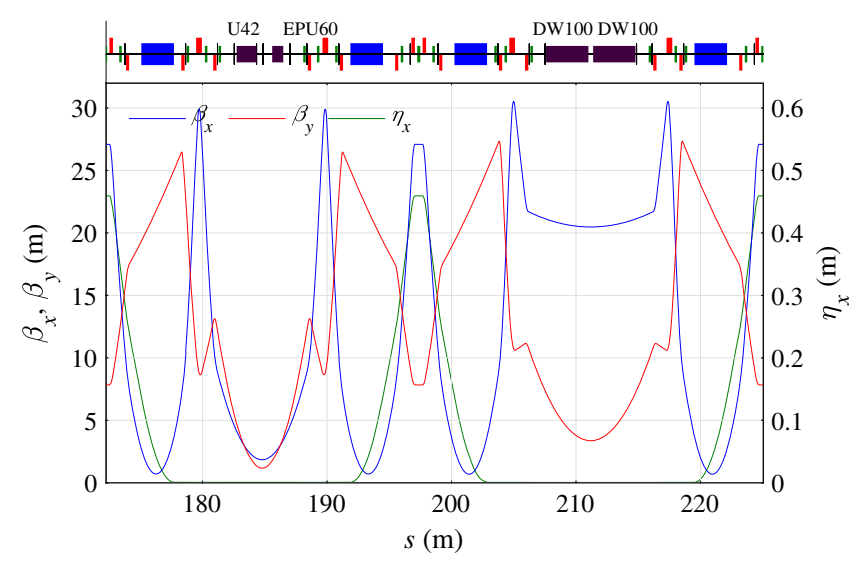

FIG. 1. Beta functions for 1/15 of NSLS-II (cells 7 and 8). 
TABLE I. NSLS-II storage ring.

\begin{tabular}{lc}
\hline \hline Parameter & Value \\
\hline Energy & $3 \mathrm{GeV}$ \\
Circumference & $792 \mathrm{~m}$ \\
Horizontal emittance (bare/3DW) & $2.1 \mathrm{~nm} / 0.95 \mathrm{~nm}$ \\
Betatron tunes, hor/ver & $33.22 / 16.26$ \\
Natural chromaticity, hor/ver & $-98 /-41$ \\
Momentum compaction & $3.6 \times 10^{-4}$ \\
Energy loss per turn (bare/3DW) & $286 \mathrm{keV} / 649 \mathrm{keV}$ \\
Relative energy spread (bare/3DW) & $5.1 \times 10^{-4} / 8.2 \times 10^{-4}$ \\
Bunch length $^{\mathrm{a}}$ (bare/3DW) & $2.7 \mathrm{~mm} / 4.3 \mathrm{~mm}$ \\
\hline \hline
\end{tabular}

${ }^{\mathrm{a}}$ Zero beam current, $3 \mathrm{MV}$ RF voltage.

Code [2]. The lattice functions are calculated using ELEGANT [3] with imported kickmaps for all IDs. The relative distortion of the beta functions caused by the undulators is presented in Fig. 2. The horizontal betatron tune is shifted by about -0.01 and the vertical tune by about 0.04 .

For every ID, the lattice distortion has been locally compensated by six nearby quadrupoles to keep the beta functions unchanged in the rest of the ring. Optimization packages in ELEGANT were used for the lattice correction. The result is shown in Fig. 3, where the relative distortion of the beta functions is presented in the upper plot and the lower plot shows the relative changes of the normalized quadrupole strength $K_{1}=\frac{1}{B \rho} \frac{\partial B_{y}}{\partial x}$.

The new lattice model with all IDs has been tested experimentally. The linear lattice is characterized using a

TABLE II. NSLS-II wigglers and undulators.

\begin{tabular}{lcclcccc}
\hline \hline Cell & Beam-line & Year & Type & $L(\mathrm{~m})$ & $\begin{array}{c}\lambda_{p} \\
(\mathrm{~mm})\end{array}$ & $K^{\mathrm{a}}$ & $\begin{array}{c}\text { Gap } \\
(\mathrm{mm})\end{array}$ \\
\hline C08D & ISS & 2015 & DW100 & $2 \times 3.4$ & 100 & 16.5 & 15 \\
C18D & FXI & 2015 & DW100 & $2 \times 3.4$ & 100 & 16.5 & 15 \\
C28D & XPD & 2015 & DW100 & $2 \times 3.4$ & 100 & 16.5 & 15 \\
C02C & SIX & 2017 & EPU57 & 3.5 & 57 & 4.4 & 16 \\
C03C & HXN & 2015 & IVU20 & 3 & 20 & 1.83 & 5.2 \\
C04U & ISR & 2016 & IVU23 & 2.8 & 23 & 2.05 & 6.2 \\
C05D & SRX & 2015 & IVU21 & 1.5 & 21 & 1.79 & 6.4 \\
C07U & SST1 & 2018 & OVU42 & 1.6 & 42 & 3.27 & 11.5 \\
C07D & SST2 & 2018 & EPU60 & 0.89 & 60 & 5.7 & 11.5 \\
C10C & IXS & 2015 & IVU22 & 3 & 22 & 1.56 & 7.4 \\
C11C & CHX & 2015 & IVU20 & 3 & 20 & 1.83 & 5.2 \\
C12D & SMI & 2017 & IVU23 & 2.8 & 23 & 2.05 & 6.2 \\
C16C & LIX & 2016 & IVU23 & 2.8 & 23 & 2.2 & 5.7 \\
C17U & FMX & 2016 & IVU21 & 1.5 & 21 & 1.79 & 6.4 \\
C17D & AMX & 2016 & IVU21 & 1.5 & 21 & 1.79 & 6.4 \\
C19U & NYX & 2017 & IVU18 & 1 & 18 & 1.55 & 5.6 \\
C21U & ESM & 2016 & EPU57 & 1.4 & 57 & 4.4 & 16 \\
C21D & ESM & 2016 & EPU105 & 2.7 & 105 & 11.2 & 16 \\
C23U & CSX1 & 2015 & EPU49 & 2 & 49 & 4.3 & 11.5 \\
C23D & CSX2 & 2015 & EPU49 & 2 & 49 & 4.3 & 11.5 \\
\hline \hline
\end{tabular}

${ }^{\mathrm{a}}$ For EPUs, the maximum $K$ parameter (linear mode) is shown.

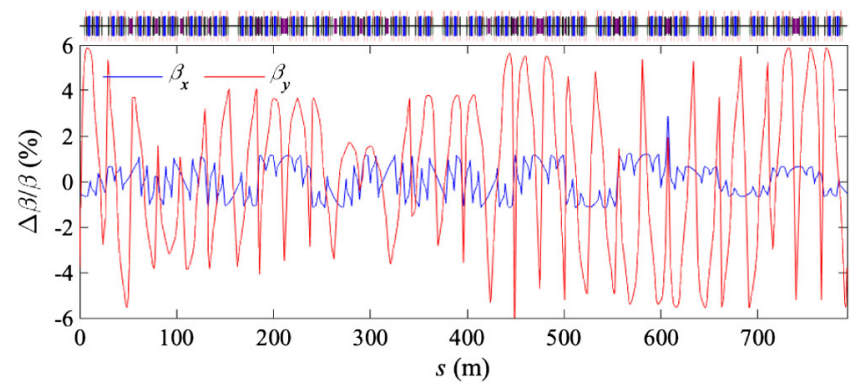

FIG. 2. Distortion of $\beta$ functions caused by the undulators.

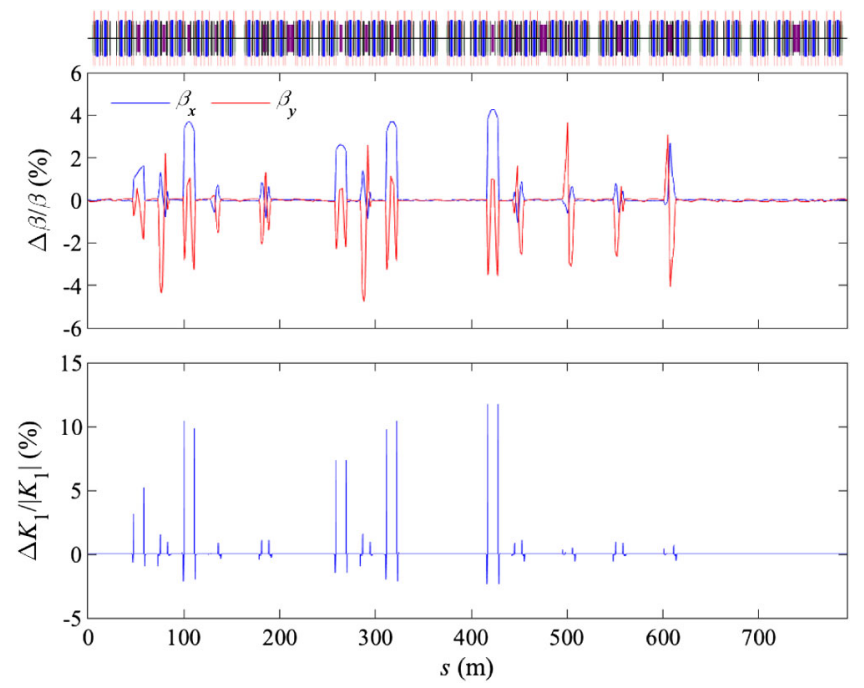

FIG. 3. Locally compensated distortion of $\beta$ functions.

technique based on the analysis of turn-by-turn data measured by beam position monitors after the betatron oscillation is excited by a pulse kicker $[4,5]$. The beam motion is controlled in the linear regime by adjusting the excitation magnitude. The method to obtain beta functions from measured turn-by-turn beam oscillations was proposed in Budker Institute [6] and realized at VEPP-4M [7,8], LEP [9], and many other accelerator facilities. Experimental crosscheck of lattice correction algorithms carried out at NSLS-II [10] shows the techniques based on measurement of turn-by-turn beam position provide almost the same correction quality as LOCO [11] based on closed orbit measurement, but they are much less time-consuming. The difference between the measured lattice functions and the design model is then corrected by tuning quadrupoles with the rigid regression developed from a Bayesian approach [12]. We have corrected the NSLS-II beta functions with the relative accuracy better than $1 \%$.

\section{EMITTANCE AND ENERGY SPREAD}

Synchrotron radiation generated by the electron beam in wigglers and undulators results in additional beam energy loss affecting the beam emittance and energy spread. The 
energy loss of an electron passing through a wiggler or undulator is given by [1]

$$
U_{\mathrm{ID}}=\frac{1}{3} r_{e} E_{e} \gamma^{2} K^{2}\left(\frac{2 \pi}{\lambda_{p}}\right)^{2} L,
$$

where $r_{e}$ is the classical electron radius, $\gamma$ is the Lorentz factor, and $K$ is the undulator strength parameter (1).

The insertion devices change the radiation integrals [13] used to calculate emittance

$$
\varepsilon_{x}=C_{q} \frac{\gamma^{2} I_{5 x}}{I_{2}-I_{4 x}}
$$

and energy spread

$$
\frac{\sigma_{E}}{E}=\sqrt{C_{q} \frac{\gamma^{2} I_{3}}{2 I_{2}+I_{4 x}+I_{4 y}}}
$$

of the electron beam. Here $I_{n}$ are the radiation integrals and $C_{q}=\frac{55}{32 \sqrt{3}} \frac{\hbar c}{E_{e}} \simeq 3.83 \times 10^{-13} \mathrm{~m}$.

For an ideal sinusoidal insertion device located in a zerodispersion section, the radiation integrals can be calculated by the simple formulas [14]:

$I_{2}=\frac{L}{2 \rho^{2}}, \quad I_{3}=\frac{4 L}{3 \pi \rho^{3}}, \quad I_{5 x} \simeq \frac{\lambda_{p}^{2}}{15 \pi^{3} \rho^{5}}\left\langle\beta_{x}\right\rangle L$,

where $\rho$ is the peak bending radius of the ID field and $\left\langle\beta_{x}\right\rangle$ is the horizontal beta function averaged along the device.

\section{BEAM-BASED MEASUREMENTS}

We measured the beam emittance, energy spread, and radiation energy loss for three modes of the NSLS-II lattice: 1. Bare lattice (no wigglers and undulators); 2. Lattice with damping wigglers only; 3 . Lattice with all wigglers and undulators.

Collective effects, such as potential well distortion, intrabeam scattering and coherent instabilities can affect the measured emittance, energy spread and bunch length. To minimize the collective effects, the measurements were carried out with a low beam intensity; the total beam current was $10 \mathrm{~mA}$ in 1000 bunches or $10 \mu \mathrm{A}$ per bunch.

\section{A. Emittance}

The NSLS-II emittance is routinely measured by an $x$-ray pinhole camera [15] installed in the NSLS-II diagnostic beamline. However, there is a significant systematic error depending on the beam intensity and the camera settings must be accurately adjusted before the measurement.

High-precision measurements of the beam emittance were carried out at the coherent hard x-ray (CHX) beamline using $\mathrm{x}$-ray beam generated by an in-vacuum undulator
IVU20 installed in cell 11 . The emittance values were calculated from precise measurements of intensity profiles of the focused undulator radiation in a simple electron beam imaging scheme. A monochromatic x-ray beam (7th spectral harmonic of the $3 \mathrm{~m}$ IVU20 in-vacuum undulator at $13.1 \mathrm{keV}$ photon energy) was refocused with a lens at the nominal sample position of the CHX beamline $(48.7 \mathrm{~m}$ downstream from the center of the straight section) to produce a focused spot $16.9 \mathrm{~m}$ further downstream. The lens was a $2 \mathrm{D}$ parabolic-shape Be lens with $\sim 50 \mu \mathrm{m}$ radius at the tip of parabola and $\sim 350 \mu \mathrm{m}$ geometrical aperture. The horizontal size of this focused beam was measured by scanning a chromium slide of $50 \mathrm{~nm}$ thickness (deposited atop a $\sim 200 \mu \mathrm{m}$ deep thin wall etched into a $\mathrm{Si}$ wafer) across the beam, using nanometerprecision encoded piezo stages. The fluorescent signal of the chromium layer was detected by an energy-dispersive detector, enabling measurements of the beam size with tens of nanometer precision.

The measured value of the horizontal FWHM of the focused x-ray beam was $(32.2 \pm 1.0) \mu \mathrm{m}$ with all ID gaps closed to their nominal values. The FWHM of the pointspread function, calculated with SRW [16,17], was $\sim 4.6 \mu \mathrm{m}$ (mainly determined by the lens aperture), so the measured spot size was strongly dominated by the electron beam size, i.e., by the horizontal emittance. The measured spot size corresponds to $(0.76 \pm 0.05) \mathrm{nm}$ horizontal emittance, assuming a $1.84 \mathrm{~m}$ horizontal beta function in the middle of low- $\beta$ straight section.

To compare with the measured data, the emittance was calculated using formula (3) adding the IDs one-by-one to the bare lattice. Using ELEGANT, the simulations were carried out with lattice distortions caused by every ID locally corrected. Figure 4 shows the measured data in comparison with formula (3) and ELEGANT simulations. By design, the damping wigglers have the most effect on the emittance reduction, about a factor of two. The effect of all 17 undulators is an additive $15 \%$.

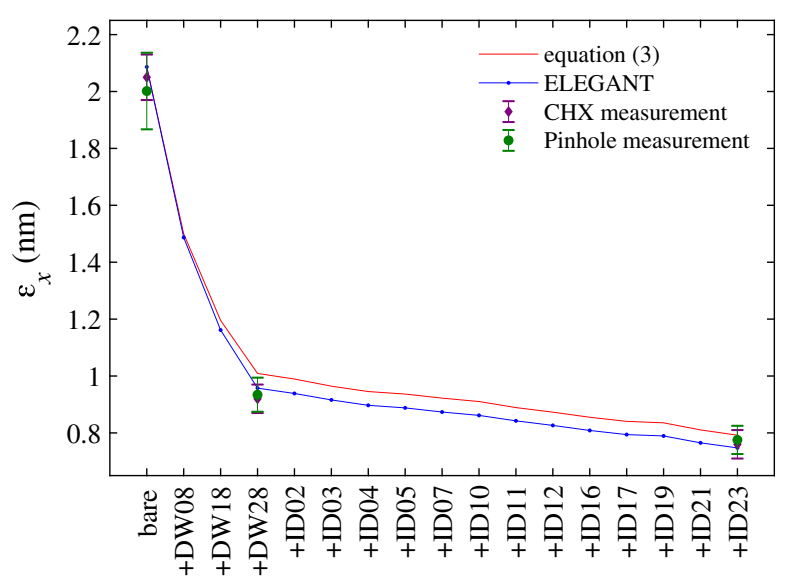

FIG. 4. Horizontal emittance vs ID number. 


\section{B. Energy loss}

The synchronous phase $\varphi_{s}$ is determined by the radiation energy loss per turn $U_{0}$ and the accelerating rf voltage $V_{\text {rf }}$. Every insertion device contributes an addition $U_{\text {ID }}$ (2) to the total energy loss resulting in the synchronous phase shift

$$
\Delta \varphi_{s}=\arcsin \frac{U_{0}}{V_{\mathrm{rf}}}-\arcsin \frac{U_{0}+U_{\mathrm{ID}}}{V_{\mathrm{rf}}} .
$$

The phase shifts caused by the damping wigglers and by 17 undulators was experimentally determined by fitting the longitudinal bunch profiles measured using a streak camera [18]. The streak camera was preliminary warmed up and the measurements were done in a minimum possible time interval to reduce the systematic error caused by the streak camera drift. The r.m.s. bunch length $\sigma_{t}$ and arrival time $t_{c}$ were calculated by Gaussian fitting the averaged 50 bunch profiles for the bare lattice, the lattice with damping wigglers, and the lattice with all IDs. The measured bunch profiles and the Gaussian fit curves are shown in Fig. 5 for each lattice. Since there is no absolute phase calibration, this technique allows us to measure only the synchronous phase difference $\Delta \varphi_{s}=\left(t_{c 2}-t_{c 1}\right) \omega_{\mathrm{rf}}$.

Solving Eq. (6), we find the ID contribution $U_{\mathrm{ID}}$ to the total energy loss. The measured data in comparison with formula (2) and with the ELEGANT simulations are presented in Fig. 6.

A factor of two increase in the beam energy loss introduced by the damping wigglers results in a factor of two emittance reduction. The equilibrium emittance is reached when quantum excitation proportional to the numerator of equation (3) is equal to radiation damping proportional to the denominator of (3). At NSLS-II, all wigglers are installed in the dispersionfree straight sections. The dispersion in the straights has been well corrected to zero and the intrinsic dispersion of the wigglers is negligible, so their contributions to the radiation integral

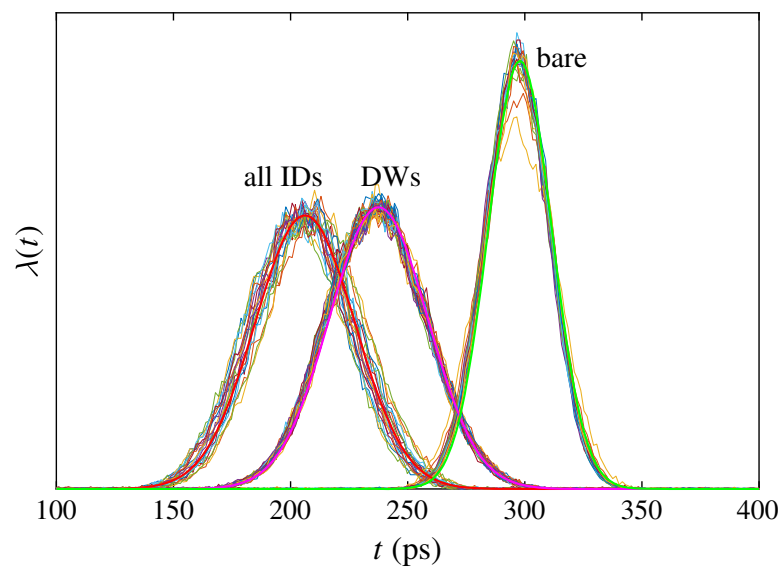

FIG. 5. Bunch profiles measured by streak camera.

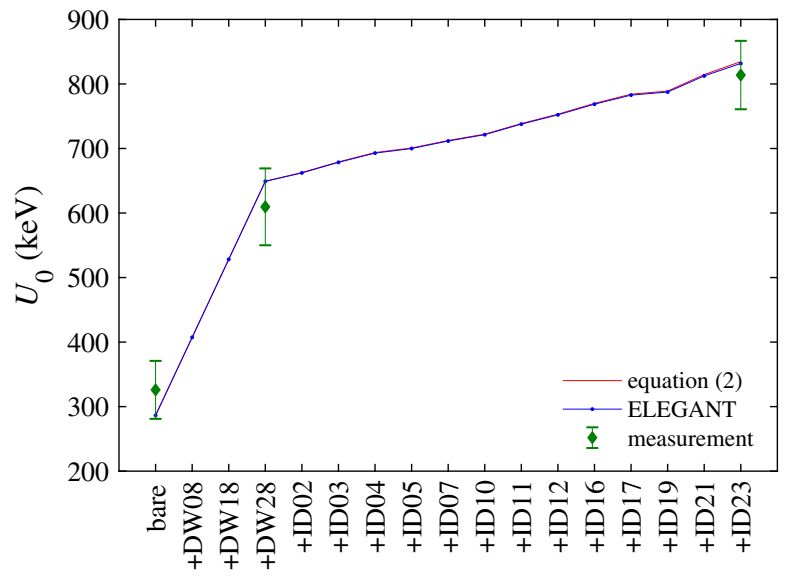

FIG. 6. Radiation energy loss vs ID number.

$I_{5}=\int \frac{\beta_{x} \eta_{x}^{\prime 2}+2 \alpha_{x} \eta_{\eta} \eta_{x}^{\prime}+\gamma_{x} \eta_{x}^{2}}{|\rho|^{3}} d s$ in the numerator of (3) and to the $I_{4}=\int \frac{\eta_{x}}{\rho^{3}}\left(1+2 \rho^{2} K_{1}\right) d s$ term in the denominator of (3) are negligible compared to the $I_{2}=\int \frac{1}{\rho^{2}} d s$ term. By having twice the beam energy loss from the wigglers, the radiation damping is doubled and the quantum excitation is not changed. Hence, the equilibrium emittance should be reduced by half.

\section{Energy spread}

Since the beam current was only $10 \mu A$ per bunch, we can neglect collective effects, such as potential well distortion and intrabeam scattering. The energy spread $\sigma_{E} / E$ can be estimated from the standard low-current bunch length formula

$$
\sigma_{t}=\frac{\sigma_{E} / E}{\omega_{0}} \sqrt{\frac{2 \pi \alpha E / e}{h_{\mathrm{rf}} V_{\mathrm{rf}} \cos \varphi_{s}}},
$$

where $h_{\mathrm{rf}}$ is the $\mathrm{rf}$ harmonic number, $\alpha$ is the momentum compaction factor. The r.m.s. bunch length $\sigma_{t}$ was obtained by a Gaussian fit of the longitudinal bunch profiles registered by the streak camera.

The relative beam energy spread was also determined from the spectral scan of 7th harmonic of IVU20 undulator radiation vs photon energy measured in projection geometry (without the x-ray beam focusing) and from SRW simulations. The spectral measurements of the 7 th harmonic were performed through a $50 \times 50 \mu \mathrm{m}^{2}$ aperture $34.3 \mathrm{~m}$ donwstream from the center of the straight section and employed the fluorescent signal from a metal foil detected by a pin diode [19]. The corresponding SRW simulations used the same geometry as the measurements and the magnetic field data from magnetic measurements of the CHX IVU. In the simulations, the IVU was assumed to be perfectly aligned with respect to the electron beam orbit. 


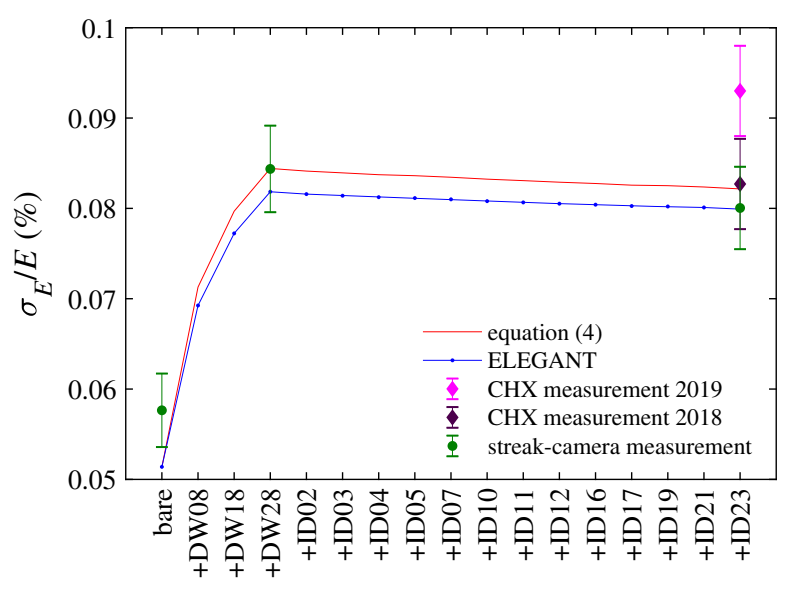

FIG. 7. Beam energy spread vs ID number.

Figure 7 shows the measured data in comparison with formula (4) and with the ELEGANT simulations. Note that, unlike the wigglers, the undulators result in a small decrease in energy spread. This effect was calculated using formula (4), simulated using ELEGANT, and confirmed by the measurements (see Fig. 7).

The energy spread measured at the CHX beamline is about $0.93 \times 10^{-3}$ with all the ID gaps closed, with a measurement error of about 5\%. The calculation does not include systematic errors, such as misalignment of IVUs relative to the electron beam or the pinhole relative to the emission axis. The result of a previous (2018) measurement [20] also shown in Fig. 7 is in better agreement with the model and steak-camera measurement. We note that the previous energy spread measurements at the CHX beamline were done after a special undulator alignment procedure [21] followed by multiple accuracy tests, which could not be accomplished before the recent measurement because of time constraints.

The observed discrepancy between the 2018 and 2019 measurements can be explained by a misalignment of the CHX IVU by a few tens of microns, which might arise due to floor movements over time. While such small misalignment may have a noticeable impact on the measured spectrum (in particular, harmonic widths and peak intensities), its real impact on the energy spread of the electron beam is negligible.

\section{CONCLUSION}

NSLS-II is a high-brightness synchrotron light source in user operations since 2014. Up to now, 20 light-generating insertion devices are installed: 3 damping wigglers and 17 undulators. The lattice distortion introduced by each insertion device is locally compensated using 6 quadrupole magnets located nearby. The lattice model of the NSLS-II storage ring with all the IDs has been developed and tested with the beam.
Beam-based measurements of the beam emittance, energy spread, and radiation energy loss were carried out using NSLS-II optical diagnostics and CHX beamline. The measurements have been done for three options of NSLS-II lattice: bare lattice (no wigglers and undulators); lattice with 3 damping wigglers only; lattice with all insertion devices. The measured emittance is $2.05 \mathrm{~nm}$, $0.92 \mathrm{~nm}$, and $0.76 \mathrm{~nm}$, respectively. The measured values of the emittance, radiation energy loss, and energy spread show a good agreement with analytical formulas and numerical simulations.

\section{ACKNOWLEDGMENTS}

The authors would like to thank Kenneth EvansLutterodt for help with the fluorescent knife edge measurements. This work has been supported by DOE under Contract No. DE-SC0012704. SRW code is partially supported by DOE SBIR Grant No. DE-SC0011237 and DOE BES Field Work Proposal grant on "New Capabilities for Simulation of Coherent X-ray Scattering Experiments at Light Sources".

[1] H. Wiedemann, Particle Accelerator Physics II (Springer, Berlin, 1999).

[2] O. Chubar, P. Elleaume, and J. Chavanne, A 3D magnetostatics computer code for insertion devices, J. Synchrotron Radiat. 5, 481 (1998).

[3] M. Borland, elegant: A flexible SDDS-compliant code for accelerator simulation, Argonne National Laboratory, Advanced Photon Source Report No. LS-287, 2000.

[4] G. Wang, T. Shaftan, F. Willeke et al., NSLS-II SR commissioning report, Brookhaven National Laboratory Report No. BNL-211142-2019-TECH, 2015, pp. 36-41, https://www.osti.gov/servlets/purl/1505098.

[5] Y. Li, L. Yang, and W. Cheng, Using big data in NSLS-II storage ring commissioning, in Proc. of ICAP-2015, Shanghai (MODBC1), http://accelconf.web.cern.ch/ AccelConf/ICAP2015/papers/modbc1.pdf.

[6] A. M. Batrakov, A. S. Kalinin, I. Y. Protopopov, and A. D. Khilchenko, Diagnostics of injected and circulating beams using pickup-electrodes at the VEPP-4 storage ring, Report No. Budker INP 80-167, Novosibirsk, 1980 (in Russian), http://www.inp.nsk.su/images/preprint/1980_167.pdf.

[7] A. N. Dubrovin, A. S. Kalinin, E. A. Simonov, V. V. Smaluk, and D. N. Shatilov, Measurement and correction of beta function at the VEPP-4M storage ring, in Proc. of RuPAC-1994, Protvino (Institute for High Energy Physics (IHEP), Protvino, Moscow Region, Russia, 1994), pp. 207-212 (in Russian), ISBN:5-88738-001-2.

[8] A. N. Dubrovin, A. S. Kalinin, D. N. Shatilov, E. A. Simonov, and V. V. Smaluk, Applications of beam diagnostic system at the VEPP-4, in Proc. of EPAC-1996, Sitges, (TUP010L), http://accelconf.web.cern.ch/ AccelConf/e96/PAPERS/TUPL/TUP010L.PDF.

[9] P. Castro, J. Borer, A. Burns, G. Morpurgo, and R. Schmidt, Betatron function measurement at LEP using 
the BOM 1000 turns facility, in Proceedings of the 15th Particle Accelerator Conference, PAC-1993, Washington, DC, 1993 (IEEE, New York, 1993), pp. 21032105.

[10] V. Smaluk, X. Yang, W. Guo, Y. Hidaka, G. Wang, Y. Li, L. Yang, Experimental crosscheck of algorithms for magnet lattice correction, in Proc. of IPAC-2016, Busan (THPMR008), http://accelconf.web.cern.ch/accelconf/ ipac2016/papers/thpmr008.pdf.

[11] J. Safranek, Experimental determination of storage ring optics using closed orbit response measurements, Nucl. Instrum. Methods Phys. Res., Sect. A 388, 27 (1997).

[12] Y. Li, R. Rainer, and W. Cheng, Bayesian approach for linear optics correction, Phys. Rev. Accel. Beams 22, 012804 (2019).

[13] R. H. Helm, M. J. Lee, P. L. Morton, and M. Sands, Evaluation of synchrotron radiation integrals, in Proc. of PAC-1973, San Francisco, pp. 900-901, http://accelconf .web.cern.ch/AccelConf/p73/PDF/PAC1973_0900.PDF.

[14] S. Y. Lee, Accelerator Physics (World Scientific, Singapore, 1999).

[15] W. Cheng, B. Bacha, B. Kosciuk, and D. Padrazo, Improvements of NSLS-II x-ray diagnostics beamlines, in Proc. of IPAC-2018, Vancouver (WEPAF012), https:// accelconf.web.cern.ch/AccelConf/ipac2018/papers/ wepaf012.pdf.

[16] O. Chubar and P. Elleaume, Accurate and efficient computation of synchrotron radiation in the near field region, in Proceedings of the 6th European Particle Accelerator
Conference, Stockholm, 1998 (IOP, London, 1998), pp. 1177-1179.

[17] T. Weitkamp, O. Chubar, M. Drakopoulos, A. Souvorov, I. Snigireva, A. Snigirev, F. Günzler, C. Schroer, and B. Lengeler, Refractive lenses as a beam diagnostics tool for high-energy synchrotron radiation, Nucl. Instrum. Methods Phys. Res., Sect. A 467-468, 248 (2001).

[18] W. Cheng, B. Bacha, A. Blednykh, Y. Li, and O. Singh, Longitudinal bunch profile measurement at NSLS2 storage ring, in Proc. of IBIC-2015, Melbourne (MOPB083), http://accelconf.web.cern.ch/AccelConf/ IBIC2015/papers/mopb083.pdf.

[19] L. Wiegart, M. Rakitin, A. Fluerasu, and O. Chubar, X-ray optical simulations supporting advanced commissioning of the coherent hard X-ray beamline at NSLS-II, in Proc. of SPIE 10388, Advances in Computational Methods for X-Ray Optics IV, 103880N (2017), https://doi.org/ 10.1117/12.2274403.

[20] A. Blednykh, B. Bacha, G. Bassi, W. Cheng, O. Chubar, A. Derbenev, R. Lindberg, M. Rakitin, V. Smaluk, M. Zhernenkov, Y.-C. K. Chen-Wiegart, and L. Wiegart, New aspects of longitudinal instabilities in electron storage rings, Sci. Rep. 8, 11918 (2018).

[21] O. Chubar, C. Kitegi, Y.-C. K. Chen-Wiegart, D. Hidas, Y. Hidaka, T. Tanabe, G. Williams, J. Thieme, T. Caswell, M. Rakitin, L. Wiegart, A. Fluerasu, L. Yang, S. Chodankar, and M. Zhernenkov, Spectrum-based alignment of invacuum undulators in a low-emittance storage ring, Synchrotron Radiat. News 31, 4 (2018). 